\title{
Selective-Area-Grown Semiconductor-Superconductor Hybrids: A Basis for Topological Networks
}

\author{
S. Vaitiekenas, ${ }^{1}$ A. M. Whiticar, ${ }^{1}$ M.-T. Deng, ${ }^{1}$ F. Krizek, ${ }^{1}$ J. E. Sestoft, ${ }^{1}$ C. J. Palmstrøm, ${ }^{2}$ \\ S. Marti-Sanchez, ${ }^{3}$ J. Arbiol, ${ }^{3,4}$ P. Krogstrup, ${ }^{1}$ L. Casparis, ${ }^{1}$ and C. M. Marcus ${ }^{1}$ \\ ${ }^{1}$ Center for Quantum Devices and Station $Q$ Copenhagen, Niels Bohr Institute, \\ University of Copenhagen, Universitetsparken 5, 2100 Copenhagen, Denmark \\ ${ }^{2}$ Materials Department, University of California, Santa Barbara, California 93106, USA \\ ${ }^{3}$ Catalan Institute of Nanoscience and Nanotechnology (ICN2), CSIC and BIST, Campus UAB, 08193 Bellaterra, \\ Barcelona, Spain \\ ${ }^{4}$ ICREA, Pg. Lluís Companys 23, 08010 Barcelona, Catalonia, Spain
}

(Received 18 March 2018; revised manuscript received 20 June 2018; published 3 October 2018)

\begin{abstract}
We introduce selective area grown hybrid InAs/Al nanowires based on molecular beam epitaxy, allowing arbitrary semiconductor-superconductor networks containing loops and branches. Transport reveals a hard induced gap and unpoisoned $2 e$-periodic Coulomb blockade, with temperature dependent $1 e$ features in agreement with theory. Coulomb peak spacing in parallel magnetic field displays overshoot, indicating an oscillating discrete near-zero subgap state consistent with device length. Finally, we investigate a loop network, finding strong spin-orbit coupling and a coherence length of several microns. These results demonstrate the potential of this platform for scalable topological networks among other applications.
\end{abstract}

DOI: 10.1103/PhysRevLett.121.147701

Majorana zero modes (MZMs) at the ends of onedimensional topological superconductors are expected to exhibit non-Abelian braiding statistics [1,2], providing naturally fault-tolerant qubits $[3,4]$. Proposed realizations of braiding [5,6], interference-based topological qubits [7-9], and topological quantum computing architectures [10] require scalable nanowire networks. While relatively simple branched or looped wires can be realized by specialized growth methods [11,12] or by etch- and gate-confined two-dimensional hybrid heterostructures [13-16], selective area growth (SAG) [17] enables deterministic patterning of arbitrarily complex structures. This allows complex continuous patterns of superconductorsemiconductor hybrids and topological networks.

Following initial theoretical proposals [18,19], a number of experiments have reported signatures of MZMs in hybrid semiconductor-superconductor nanowires [11], including zero-bias conductance peaks $[15,16,20-26]$ and Coulomb blockade peak spacing oscillations $[27,28]$. To date, experiments have used individual vapor-liquid-solid (VLS) nanowires [20-25] or gate-confined two-dimensional heterostructures $[15,16]$. Within these approaches, constructing complex topological devices and networks containing branches and loops [5-10] is a challenge. Recently, branched and looped VLS growth has been developed toward this goal $[12,29]$.

In this Letter, we investigate a novel approach to the growth of semiconductor-superconductor hybrids that allows deterministic on-chip patterning of topological superconducting networks based on SAG. We characterize key physical properties required for building Majorana networks, including a hard superconducting gap, induced in the semiconductor, phase-coherence length of several microns, strong spin-orbit coupling, and Coulomb blockade peak motion compatible with interacting Majoranas. Overall, these properties show great promise for SAGbased topological networks.

Selective area growth was realized on a semi-insulating (001) InP substrate. PECVD grown $\mathrm{SiO}_{\mathrm{x}}$ was patterned using electron beam lithography and wet etching. InAs wires with triangular cross sections were grown by molecular beam epitaxy (MBE). The $\mathrm{Al}$ was grown in situ by MBE using angled deposition covering one of the facets. The excess Al was removed by wet etching [Figs. 1(a)1(c)]. The details of the semiconductor growth are given in Ref. [17], while it is superconductor-semiconductor proximity effects that are emphasized in the present study. Data from four devices are presented. Device 1 [Fig. 1(d)] consists of a single barrier at the end of a $4 \mu \mathrm{m}$ wire, defined by a lithographically patterned gate adjacent to a $\mathrm{Ti} / \mathrm{Au}$ contact where the $\mathrm{Al}$ has been removed by wet etching. This device allowed density of states measurement at the end of the wire by means of bias spectroscopy, to investigate the superconducting proximity effect in the InAs. Evolution of Coulomb blockade in temperature and magnetic field was studied in device 2 [Fig. 2(b)] — a hybrid quantum dot with length of $1.1 \mu \mathrm{m}$ defined by two Ti/Au gates adjacent to etched-Al regions. The barrier voltages 
(a)

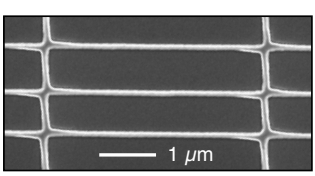

(c)

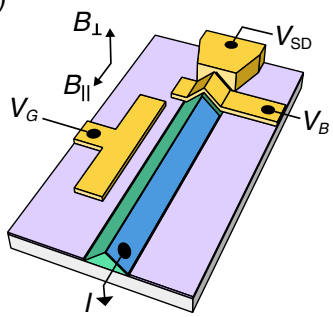

(b)

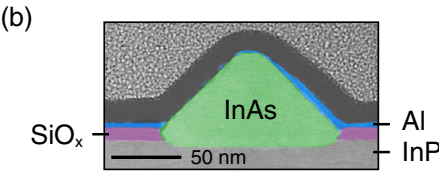

(d)

(e)
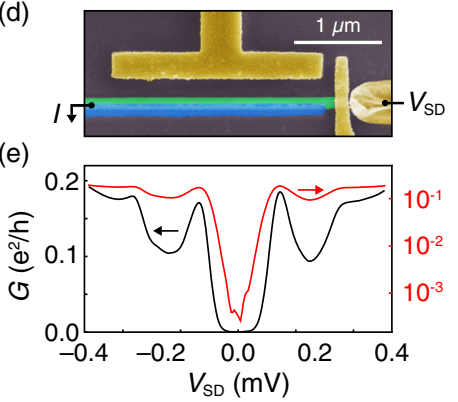

FIG. 1. (a) Scanning electron micrograph of a SAG hybrid network. (b) False-colored annular dark field scanning transmission electron micrograph of a nanowire cross section displays InP substrate, InAs (green) nanowire, $\mathrm{Al}$ (blue) shell, and $\mathrm{SiO}_{\mathrm{x}}$ (purple) mask. (c) Measurement set-up for device 1 showing the gate voltages and orientations of magnetic fields used in the measurements. (d) False-colored electron micrograph of device 1. (e) Differential conductance, $G$, as a function of source-drain bias, $V_{\mathrm{SD}}$, in linear (black) and logarithmic (red) scales, shows a hard superconducting gap.

$V_{B}$ were used to create tunneling barriers. The chemical potential in the wires was tuned with gate voltage $V_{G}$. Device 3 was a micron-size square loop [Fig. 4(a)] with fully removed $\mathrm{Al}$, which was used to extract phase
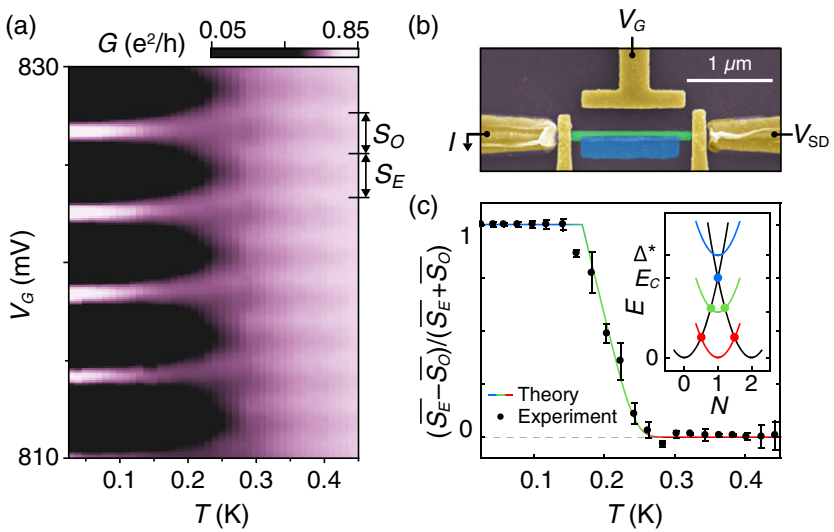

FIG. 2. (a) Conductance, $G$, of device 2 as a function of applied gate voltage, $V_{G}$, and temperature, $T$. A characteristic $2 e$ to $1 e$ transition occurs around $200 \mathrm{mK}$. The color scale was adjusted for better visibility. (b) False-colored electron micrograph of device 2. (c) Normalized even-odd peak spacing difference, $\left(\overline{S_{E}}-\overline{S_{O}}\right) /\left(\overline{S_{E}}+\overline{S_{O}}\right)$, from the measurements shown in (b) as a function of $T$. The error bars were estimated using standard deviation of the peak spacing. The theoretical fit corresponds to an induced superconducting gap $\Delta^{*}=190 \mu \mathrm{eV}$. Inset: Energy, $E$, of the device as a function of normalized gate voltage, $N$. Black (color) parabolas correspond to the even(odd)-parity ground state. Transport occurs at the charge degeneracy points indicated by filled circles.

coherence lengths from weak antilocalization (WAL) and Aharonov-Bohm (AB) oscillations. Device 4 (Fig. S3 in Supplemental Material [30]) — a top-gated nanowire without $\mathrm{Al}$ - was used to extract the charge carrier mobility. The nanowires in devices 1, 2, and 4 are parallel to [100] direction, whereas the arms of device 3 are oriented along [100] and [010] directions. Standard ac lock-in measurements were carried out in a dilution refrigerator with a three-axis vector magnet. See Supplemental Material [30] for more detailed description of the growth, fabrication and measurement setup.

Differential conductance, $G$, in the tunneling regime, as a function of source-drain bias, $V_{\mathrm{SD}}$, for device 1 [Fig. 1(e)] at $V_{G}=-9.2 \mathrm{~V}$ reveals a gapped density of states with two peaks at $V_{\mathrm{SD}}=110 \mu \mathrm{eV}$ and $280 \mu \mathrm{eV}$. We tentatively identify the two peaks with two populations of carriers in the semiconductor, the one with a larger gap residing at the InAs-Al interface and with a smaller at the InAs-InP. The magnitude of the larger superconducting gap is consistent with enhanced energy gaps of $290 \mu \mathrm{eV}$ for a $7 \mathrm{~nm}$ Al film [34]. The zero-bias conductance is $\sim 400$ times lower than the above-gap conductance, a ratio exceeding VLS nanowire [12,35,36] and 2DEG devices [14], indicating a hard induced gap. We note, however, that cotunneling through a quantum-dot or multichannel tunneling can enhance this ratio [37]. The spectrum evolution with $V_{G}$ from enhanced to suppressed conductance around $V_{\mathrm{SD}}=0 \mathrm{mV}$ is shown in Fig. S1 in Supplemental Material [30].

Transport through a Coulomb island geometry [Fig. 2] at low temperatures shows $2 e$-periodic peak spacing as a function of $V_{G}$. Coulomb diamonds at finite bias yield a charging energy $E_{C}=60 \mu \mathrm{eV}$ (see Fig. S2 in Supplemental Material [30]), smaller than the induced gap, $\Delta^{*} \sim 100 \mu \mathrm{eV}$, as seen in Fig. 1(e). The zero-bias Coulomb blockade spacing evolves to even-odd and finally to $1 e$-periodic peaks with increasing temperature, $T$. The $2 e$ to $1 e$ transition in temperature does not result from the destruction of superconductivity, but rather arises due to the thermal excitation of quasiparticles on the island, as investigated previously in metallic islands [38,39] and semiconductor-superconductor VLS nanowires [40].

A thermodynamic analysis of Coulomb blockade peak spacings is based on the difference in free energies, $F=F_{O}-F_{E}$, between even and odd occupied states. We consider a simple model that assumes a single induced gap $\Delta^{*}$, not accounting for the double-peaked density of states in Fig. 1(e). At low temperatures $\left(T \ll E_{C}, \Delta^{*}\right), F$ approaches $\Delta^{*}$. Above a characteristic poisoning temperature, $T_{p}$, quasiparticles become thermally activated and $F$ decreases rapidly to zero. For $F(T)>E_{C}$, Coulomb peaks are $2 e$ periodic with even peak spacings, $S_{E} \propto E_{C}$, independent of $T$. For $F(T)<E_{C}$, odd states become occupied, and the difference in peak spacing, $S_{E}-S_{O}$, decreases roughly proportional to $F$. A full analysis following Ref. [40] (see Supplemental Material [30]) yields the peak spacing difference 


$$
\begin{aligned}
S_{E}-S_{O} & =\frac{2}{\eta e} \min \left(E_{C}, F\right) \\
& =\left(S_{E}+S_{O}\right) \min \left(1, F / E_{C}\right),
\end{aligned}
$$

where $\eta$ is the dimensionless gate lever arm measured from Coulomb diamonds.

Figure 2(c) shows the measured even-odd difference in peak spacing, $\left(\overline{S_{E}}-\overline{S_{O}}\right) /\left(\overline{S_{E}}+\overline{S_{O}}\right)$, averaged over four peaks in device 2, along with Eq. (1). Thermodynamic analysis shows an excellent agreement with the peak spacing data across the full range of temperatures. The fit uses an independently measured $E_{C}$, with the induced gap as a single fit parameter, yielding $\Delta^{*}=190 \mu \mathrm{eV}$, a reasonable value that lies between the two density of states features in Fig. 1(e). The island remains unpoisoned below $T_{p} \sim 250 \mathrm{mK}$.

The evolution of Coulomb blockade peaks with parallel magnetic field, $B_{\|}$, is shown in Fig. 3(a). In this data set, peaks show even-odd periodicity at zero field due to a gatedependent gap or a bound state at energy $E_{0}$ less than $E_{C}$. A subgap state results in even-state spacing proportional to $E_{C}+E_{0}$ and odd-state spacing $E_{C}-E_{0}$ [27] (see Supplemental Material [30]), giving

$$
\begin{aligned}
S_{E, O} & =\frac{1}{\eta e}\left[E_{C} \pm \min \left(E_{C}, E_{0}\right)\right] \\
& =\frac{S_{E}+S_{O}}{2}\left[1 \pm \min \left(1, E_{0} / E_{C}\right)\right] .
\end{aligned}
$$

Figure 3(b) shows the $B_{\|}$dependence of even and odd peak spacings, $\overline{S_{E, O}} /\left(\overline{S_{E}}+\overline{S_{O}}\right)$, extracted from the data in Fig. 3(a), giving an effective $g$ factor of $\sim 13$. Even and odd peak spacings become equal at $B_{\|}=150 \mathrm{mT}$, then overshoot at higher fields with a maximum amplitude corresponding to $(7 \pm 1) \mu \mathrm{eV}$. At more positive gate voltages [Fig. 3(c)], where the carrier density is higher, peaks are $2 e$ periodic at zero field, then transition through even-odd to $1 e$-periodic Coulomb blockade without an overshoot, with an effective $g$ factor of $\sim 31$.

Overshoot of peak spacing, with $S_{O}$ exceeding $S_{E}$, indicates a discrete subgap state crossing zero energy $[27,41]$, consistent with interacting Majorana modes. The overshoot observed at more negative $V_{G}$ is quantitatively in agreement with the overshoot seen in VLS wires of comparable length [27]. The absence of the overshoot and the increase of the $g$ factor at positive $V_{G}$ is consistent with the gate-tunable carrier density in VLS wires [42].

To demonstrate fabrication and operation of a simple SAG network, we investigate the coherence of electron transport in the loop structure shown in Fig. 4(a), with the Al layer completely removed by wet etching. Conductance as a function of perpendicular magnetic field, $B_{\perp}$, shows a peak around zero magnetic field, characteristic of WAL
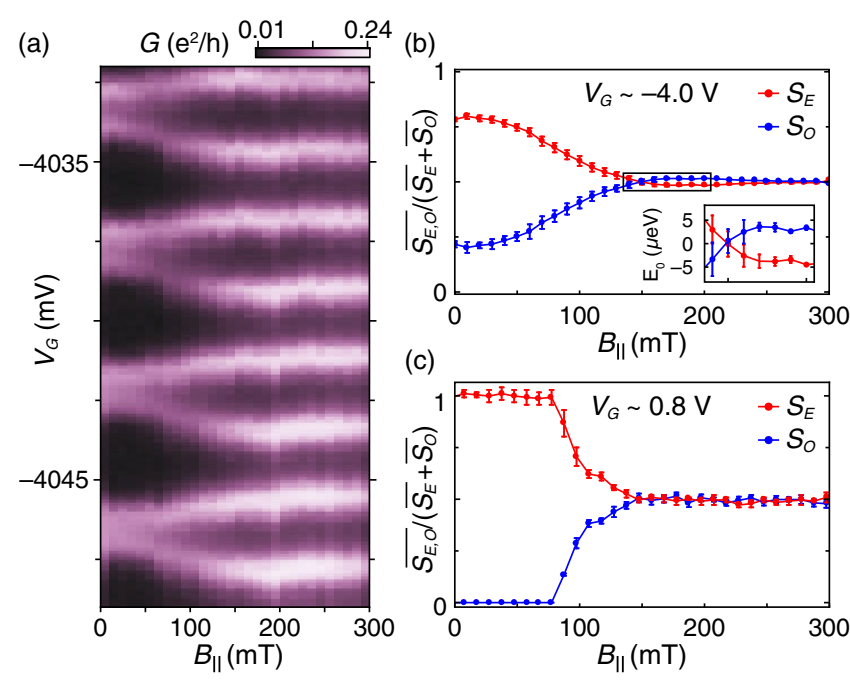

FIG. 3. (a) Conductance as a function of $V_{G}$ and $B_{\|}$from device 2 , taken at $V_{G} \sim-4.0 \mathrm{~V}$, shows even-odd peak spacings at zero field transit to $1 e$ spacing when the field is increased. (b) Normalized even and odd Coulomb peak spacings, $\overline{S_{E, O}} /\left(\overline{S_{E}}+\overline{S_{O}}\right)$, from the measurements shown in (a), as a function of $B_{\|}$. Inset: Zoom-in of the peak spacing overshoot with amplitude of $7 \mu \mathrm{eV}$ at $B_{\|}=170 \mathrm{mT}$. (c) Same as (b), but taken at $V_{G} \sim 0.8 \mathrm{~V}$. At positive gate voltage, the peaks become evenly spaced above $B_{\|}=150 \mathrm{mT}$. The error bars in (a) and (b) were estimated using the standard deviation of the peak position.

[Fig. 4(b)]. A fit to a theoretical model for disordered quasione-dimensional wires with strong spin-orbit coupling [43] yields an electron phase-coherence length $l_{\phi}^{\mathrm{WAL}} \sim 1.2 \mu \mathrm{m}$, and a spin-orbit length $l_{\mathrm{SO}} \sim 0.4 \mu \mathrm{m}$. We note that electrons propagating along [100] and [010] directions experience both Rashba and linear-Dresselhaus spin-orbit fields [44]. The magnitude of each field can be deduced from a combination of the in-plane magnetic field angle and magnitude dependence of the conductance correction due to the weak (anti-) localization. Such a study, however, is out of the scope of this work.

Upon suppressing WAL with a large perpendicular field periodic conductance oscillations are observed [Fig. 4(c)] with period $\Delta B_{\perp}=2.5 \mathrm{mT}$ corresponding to $h / e \mathrm{AB}$ oscillations with area $1.7 \mu \mathrm{m}^{2}$, matching the lithographic area of the loop. The oscillation amplitude, $A_{h / e}$, measured from the power spectral band around $h / e$ [Fig. 4(d), inset] was observed to decrease with increasing temperature as seen in Fig. 4(d). The size of $A_{h / e}$ is dictated by two characteristic lengths, thermal length $L_{T}$ and phasecoherence length $l_{\phi}^{\mathrm{AB}}$ [43]. We estimate $L_{T}$ at $20 \mathrm{mK}$ to be around $1.5 \mu \mathrm{m}$ using a charge carrier mobility $\mu=$ $700 \mathrm{~cm}^{2} /\left(\mathrm{Vs}\right.$ ) and density $n=9 \times 10^{17} \mathrm{~cm}^{-3}$ (see Supplemental Material [30]). The thermal length is comparable to the loop circumference $L=5.2 \mu \mathrm{m}$, as a result, energy averaging is expected to have finite contribution to the size of the conductance oscillations [43]. Taking 


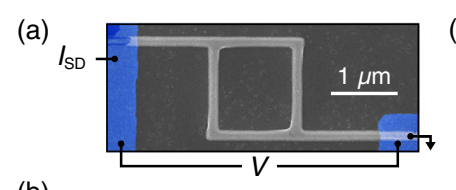

\section{(c)}
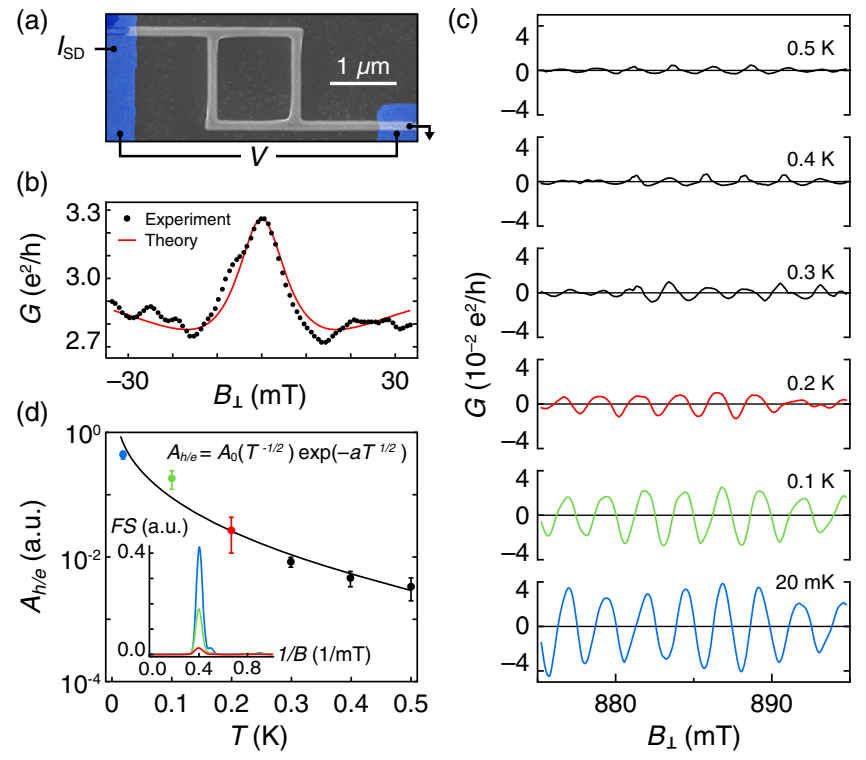

FIG. 4. (a) Electron micrograph of device 3 with false-colored epitaxial Al contacts (blue) and overlaid 4-wire measurement setup. (b) Conductance, $G$, as a function $B_{\perp}$. Red curve is a theoretical magnetoconductance displaying the weak antilocalization effect in a system with spin-orbit length, $l_{\mathrm{SO}} \sim 0.4 \mu \mathrm{m}$, and phase-coherence length, $l_{\phi}^{\mathrm{WAL}} \sim 1.2 \mu \mathrm{m}$. (c) Aharonov-Bohm oscillations around $B_{\perp} \sim 900 \mathrm{mT}$ at different temperatures. (d) Amplitude of the $h / e$ oscillations as a function of $T$. The exponential fit corresponds to the base-temperature phase-coherence length of $l_{\phi}^{\mathrm{AB}}=3.9 \mu \mathrm{m}$. The error bars correspond to the standard deviation between 4 data sets. Inset: Fourier spectra, $F S$, of the interference signal at $20 \mathrm{mK}$ (blue), $100 \mathrm{mK}$ (green), and $200 \mathrm{mK}$ (red) from the measurements shown in (b).

$A_{h / e} \propto L_{T}(T) \exp \left[-L / l_{\phi}^{\mathrm{AB}}(T)\right]$ with $L_{T} \propto T^{-1 / 2}$ and $l_{\phi}^{\mathrm{AB}} \propto$ $T^{-1 / 2}$ for a diffusive ring [45], a fit of the logarithmic amplitude $\log \left(A_{h / e}\right)=\log \left(A_{0}\right)-\frac{1}{2} \log T-a T^{1 / 2}$ yields $\log \left(A_{0}\right) \sim-1.5$ and $a \sim 6.7 \mathrm{~K}^{-1 / 2}$ [Fig. 4(d)], giving a base-temperature phase-coherence length $l_{\phi}^{\mathrm{AB}}(20 \mathrm{mK}) \sim$ $5.5 \mu \mathrm{m}$.

The discrepancy between the extracted $l_{\phi}^{\mathrm{WAL}}$ and $l_{\phi}^{\mathrm{AB}}$ has previously been observed in an experiment on GaAs/ AlGaAs-based arrays of micron-sized loops [46]. It has been argued theoretically that WAL and $\mathrm{AB}$ interference processes are governed by different dephasing mechanisms [45]. As a result, $l_{\phi}^{\mathrm{WAL}}$ and $l_{\phi}^{\mathrm{AB}}$ have different temperature dependences.

Our results show that selective area grown hybrid nanowires are a promising platform for scalable Majorana networks exhibiting strong proximity effect. The hard induced superconducting gap and $2 e$-periodic Coulomb oscillations imply strongly suppressed quasiparticle poisoning. The overshoot of Coulomb peak spacing in a parallel magnetic field indicates the presence of a discrete low-energy state. Despite the relatively low charge carrier mobility, the measured SAG-based network exhibits strong spin-orbit coupling and phase-coherent transport. Furthermore, the ability to design hybrid wire planar structures containing many branches and loops-a requirement for realizing topological quantum information processing - is readily achievable in SAG. Future work on SAG-based hybrid networks will focus on spectroscopy, correlations, interferometry, and manipulation of MZMs.

We thank D. Carrad, A. Higginbotham, and R. Lutchyn for valuable discussions as well as R. McNeil, C. Sørensen, and S. Upadhyay for contributions to material growth and device fabrication. The research was supported by Microsoft, the Danish National Research Foundation, and the European Commission. C. M. M. acknowledges support from the Villum Foundation. M. T. D. acknowledges support from State Key Laboratory of High Performance Computing, China. S. M.-S. acknowledges funding from "Programa Internacional de Becas 'la Caixa'Severo Ochoa." ICN2 acknowledges support from the Severo Ochoa Programme (MINECO, Grant No. SEV2013-0295) and is funded by the CERCA Programme/ Generalitat de Catalunya. Part of the present work has been performed in the framework of Universitat Autònoma de Barcelona Materials Science Ph.D. program.

[1] A. Y. Kitaev, Ann. Phys. (Amsterdam) 303, 2 (2003).

[2] N. Read and D. Green, Phys. Rev. B 61, 10267 (2000).

[3] C. Nayak, S. H. Simon, A. Stern, M. Freedman, and S. Das Sarma, Rev. Mod. Phys. 80, 1083 (2008).

[4] S. Das Sarma, M. Freedman, and C. Nayak, npj Quantum Inf. 1, 15001 (2015).

[5] J. Alicea, Y. Oreg, G. Refael, F. von Oppen, and M. P. A. Fisher, Nat. Phys. 7, 412 (2011).

[6] D. Aasen, M. Hell, R. V. Mishmash, A. Higginbotham, J. Danon, M. Leijnse, T. S. Jespersen, J. A. Folk, C. M. Marcus, K. Flensberg, and J. Alicea, Phys. Rev. X 6, 031016 (2016).

[7] K. Flensberg, Phys. Rev. Lett. 106, 090503 (2011).

[8] S. Plugge, A. Rasmussen, R. Egger, and K. Flensberg, New J. Phys. 19, 012001 (2017).

[9] S. Vijay and L. Fu, Phys. Rev. B 94, 235446 (2016).

[10] T. Karzig, C. Knapp, R. M. Lutchyn, P. Bonderson, M. B. Hastings, C. Nayak, J. Alicea, K. Flensberg, S. Plugge, Y. Oreg, C. M. Marcus, and M. H. Freedman, Phys. Rev. B 95, 235305 (2017).

[11] P. Krogstrup, N. L. B. Ziino, W. Chang, S. M. Albrecht, M. H. Madsen, E. Johnson, J. Nygård, C. M. Marcus, and T. S. Jespersen, Nat. Mater. 14, 400 (2015).

[12] S. Gazibegovic, D. Car, H. Zhang, S. C. Balk, J. A. Logan, M. W. A. de Moor, M. C. Cassidy, R. Schmits, D. Xu, G. Wang, P. Krogstrup, R. L. M. Op het Veld, K. Zuo, Y. Vos, J. Shen, D. Bouman, B. Shojaei, D. Pennachio, J. S. Lee, and P. J. van Veldhoven et al., Nature (London) 548, 434 (2017).

[13] J. Shabani, M. Kjaergaard, H. J. Suominen, Y. Kim, F. Nichele, K. Pakrouski, T. Stankevic, R. M. Lutchyn, P. Krogstrup, R. Feidenhans'l, S. Kraemer, C. Nayak, 
M. Troyer, C. M. Marcus, and C. J. Palmstrøm, Phys. Rev. B 93, 155402 (2016).

[14] M. Kjaergaard, F. Nichele, H. J. Suominen, M. P. Nowak, M. Wimmer, A. R. Akhmerov, J. A. Folk, K. Flensberg, J. Shabani, C. J. Palmstrøm, and C. M. Marcus, Nat. Commun. 7, 12841 (2016).

[15] H. J. Suominen, M. Kjaergaard, A. R. Hamilton, J. Shabani, C. J. Palmstrøm, C. M. Marcus, and F. Nichele, Phys. Rev. Lett. 119, 176805 (2017).

[16] F. Nichele, A.C.C. Drachmann, A. M. Whiticar, E. C. T. O'Farrell, H. J. Suominen, A. Fornieri, T. Wang, G. C. Gardner, C. Thomas, A. T. Hatke, P. Krogstrup, M. J. Manfra, K. Flensberg, and C. M. Marcus, Phys. Rev. Lett. 119, 136803 (2017).

[17] F. Krizek, J. E. Sestoft, P. Aseev, S. Marti-Sanchez, S. Vaitiekenas, L. Casparis, S. A. Khan, Y. Liu, T. Stankevič, A. M. Whiticar, A. Fursina, F. Boekhout, R. Koops, E. Uccelli, L. P. Kouwenhoven, C. M. Marcus, J. Arbiol, and P. Krogstrup, Phys. Rev. Mater. 2, 093401 (2018).

[18] R. M. Lutchyn, J. D. Sau, and S. Das Sarma, Phys. Rev. Lett. 105, 077001 (2010).

[19] Y. Oreg, G. Refael, and F. von Oppen, Phys. Rev. Lett. 105, 177002 (2010).

[20] V. Mourik, K. Zuo, S. M. Frolov, S. R. Plissard, E. P. A. M. Bakkers, and L.P. Kouwenhoven, Science 336, 1003 (2012).

[21] M. T. Deng, C. L. Yu, G. Y. Huang, M. Larsson, P. Caroff, and H. Q. Xu, Nano Lett. 12, 6414 (2012).

[22] A. Das, Y. Ronen, Y. Most, Y. Oreg, M. Heiblum, and H. Shtrikman, Nat. Phys. 8, 887 (2012).

[23] Ö. Gül, H. Zhang, J. D. S. Bommer, M. W. A. de Moor, D. Car, S. R. Plissard, E. P. A. M. Bakkers, A. Geresdi, K. Watanabe, T. Taniguchi, and L. P. Kouwenhoven, Nat. Nanotechnol. 13, 192 (2018).

[24] M. T. Deng, S. Vaitiekènas, E. B. Hansen, J. Danon, M. Leijnse, K. Flensberg, J. Nygård, P. Krogstrup, and C. M. Marcus, Science 354, 1557 (2016).

[25] H. Zhang, C.-X. Liu, S. Gazibegovic, D. Xu, J. A. Logan, G. Wang, N. van Loo, J. D. S. Bommer, M. W. A. de Moor, D. Car, R. L. M. Op het Veld, P. J. van Veldhoven, S. Koelling, M. A. Verheijen, M. Pendharkar, D. J. Pennachio, B. Shojaei, J. S. Lee, C. J. Palmstrøm, and E. P. A. M. Bakkers et al., Nature (London) 556, 74 (2018).

[26] J. E. Sestoft, T. Kanne, A. N. Gejl, M. von Soosten, J. S. Yodh, D. Sherman, B. Tarasinski, M. Wimmer, E. Johnson, M.-T. Deng, J. Nygård, T. S. Jespersen, C. M. Marcus, and P. Krogstrup, Phys. Rev. Mater. 2, 044202 (2018).

[27] S. M. Albrecht, A. P. Higginbotham, M. Madsen, F. Kuemmeth, T. S. Jespersen, J. Nygård, P. Krogstrup, and C. M. Marcus, Nature (London) 531, 206 (2016).
[28] D. Sherman, J. S. Yodh, S. M. Albrecht, J. Nygård, P. Krogstrup, and C. M. Marcus, Nat. Nanotechnol. 12, 212 (2017).

[29] F. Krizek, T. Kanne, D. Razmadze, E. Johnson, J. Nygård, C. M. Marcus, and P. Krogstrup, Nano Lett. 17, 6090 (2017).

[30] See Supplemental Material at http://link.aps.org/ supplemental/10.1103/PhysRevLett.121.147701 for more detailed device description as well as additional analysis and measurements, which includes Refs. [31-33].

[31] G. E. Blonder, M. Tinkham, and T. M. Klapwijk, Phys. Rev. B 25, 4515 (1982).

[32] Ö. Gül, D. J. van Woerkom, I. van Weperen, D. Car, S. R. Plissard, E. P. A. M. Bakkers, and L. P. Kouwenhoven, Nanotechnology 26, 215202 (2015).

[33] T. S. Jespersen, M. L. Polianski, C. B. Sørensen, K. Flensberg, and J Nygård. New J. Phys. 11, 113025 (2009).

[34] N. A. Court, A. J. Ferguson, and R. G. Clark, Supercond. Sci. Technol. 21, 015013 (2008).

[35] W. Chang, S. M. Albrecht, T. S. Jespersen, F. Kuemmeth, P. Krogstrup, J. Nygård, and C. M. Marcus, Nat. Nanotechnol. 10, 232 (2015).

[36] H. Zhang, Ö. Gül, S. Conesa-Boj, M. P. Nowak, M. Wimmer, K. Zuo, V. Mourik, F. K. de Vries, J. van Veen, M. W. A. de Moor, J. D. S. Bommer, D. J. van Woerkom, D. Car, S. R. Plissard, E. P. A. M. Bakkers, M. Quintero-Pérez, M. C. Cassidy, S. Koelling, S. Goswami, and K. Watanabe et al., Nat. Commun. 8, 16025 (2017).

[37] C. W. J. Beenakker, Phys. Rev. B 46, 12841 (1992).

[38] M. T. Tuominen, J. M. Hergenrother, T. S. Tighe, and M. Tinkham, Phys. Rev. Lett. 69, 1997 (1992).

[39] P. Lafarge, P. Joyez, D. Esteve, C. Urbina, and M. H. Devoret, Phys. Rev. Lett. 70, 994 (1993).

[40] A. P. Higginbotham, S. M. Albrecht, G. Kiršanskas, W. Chang, F. Kuemmeth, P. Krogstrup, T. S. Jespersen, J. Nygård, K. Flensberg, and C. M. Marcus, Nat. Phys. 11, 1017 (2015).

[41] C.-K. Chiu, J. D. Sau, and S. Das Sarma, Phys. Rev. B 96, 054504 (2017).

[42] S. Vaitiekėnas, M.-T. Deng, J. Nygård, P. Krogstrup, and C. M. Marcus, Phys. Rev. Lett. 121, 037703 (2018).

[43] C. Kurdak, A. M. Chang, A. Chin, and T. Y. Chang, Phys. Rev. B 46, 6846 (1992).

[44] A. Sasaki, S. Nonaka, Y. Kunihashi, M. Kohda, T. Bauernfeind, T. Dollinger, and J. Nitta, Nat. Nanotechnol. 9, 703 (2014).

[45] T. Ludwig and A. D. Mirlin, Phys. Rev. B 69, 193306 (2004).

[46] M. Ferrier, A. C. H. Rowe, S. Guéron, H. Bouchiat, C. Texier, and G. Montambaux, Phys. Rev. Lett. 100, 146802 (2008). 